\title{
Schilder's Disease: Abnormal Cholesterol Retention and Accumulation in Cultivated Fibroblasts
}

\author{
Barbara K. Burton and Henry L. Nadler ${ }^{[25]}$ \\ Department of Pediatrics, Northwestern University Medical School, Division of Genetics, \\ Children's Memorial Hospital, Chicago, Illinois, USA
}

\begin{abstract}
Extract
Schilder's disease (SD) with adrenal insufficiency is a fatal X-linked recessive disorder for which the basic defect is as yet unknown. To investigate the hypothesis that Schilder's disease might be associated with a generalized defect in cholesterol metabolism, the uptake and disappearance of labeled cholesterol and cholesterol esters were studied in cultivated fibroblasts from patients with Schilder's disease, patients with familial hypercholesterolemia, and normal control subjects. Over a 10-12-day period of exposure to the labeled compound, SD fibroblasts accumulated significantly more cholesterol than did normal fibroblasts $(P<0.001)$. In addition, after a 24-hr exposure to the labeled cholesterol, the SD fibroblasts retained significantly more of the label than did control cells over a 5 -day period of study $(P<0.001)$. The retained label was still located in the cholesterol fraction on thin layer chromatography in both SD and normal cells. The total cholesterol content of SD cells when measured directly after 12 days in tissue culture was significantly greater than that of normal cells.
\end{abstract}

\section{Speculation}

The results of these studies support the concept that Schilder's disease may indeed be related to a generalized defect in cholesterol metabolism or transport. An alternative explanation for these findings is that Schilder's disease may be associated with a defect in membrane structure or turnover that is not directly related to cholesterol metabolism but can be followed in vitro by cholesterol accumulation and retention.

\section{Introduction}

Schilder's disease, also called diffuse cerebral sclerosis or sudanophilic leukodystrophy, is a heterogeneous group of fatal degenerative neurologic disorders associated with morphologic findings similar to those observed in multiple sclerosis $[2,14,17]$. One entity within this group is inherited as an X-linked recessive disorder and may be associated with adrenocortical atrophy with adrenal insufficiency of varying degrees ranging from classic Addison's disease to isolated un- responsiveness to exogenous ACTH administration $[5,7,8,16,18,19]$.

Recently, Schaumburg et al. [16] have examined the adrenal glands of male patients with Schilder's disease with and without clinically demonstrable adrenal insufficiency. In all cases studied, distinctive changes were noted in the adrenal cortex including the presence of nests of ballooned, nonsudanophilic cortical cells containing cytoplasmic aggregates of birefringent crystals. Similar findings had previously been reported in Wol- 
man's disease [12] and in aniline intoxication [10], both of which are characterized by abnormalities in cholesterol metabolism. Biochemical studies of the brain in a number of patients with Schilder's disease have revealed an absolute and relative increase in esterified cholesterol [5]. Eto and Suzuki [4] have demonstrated the presence of cholesterol esters with an abnormal fatty acid composition in the brains of patients with Schilder's disease and other disorders associated with sudanophilic demyelination. Menkes and Eviatar [13] reported the presence of an abnormal sterol with the same number of carbon atoms as cholesterol in the white matter of the brain in one patient with Schilder's disease with adrenal insufficiency.

On the basis of this evidence, we hypothesized that Schilder's disease might be related to a generalized defect in cholesterol metabolism and that such a defect might be demonstrable in tissue culture. We present here the results of our investigations of the handling of labeled cholesterol and cholesterol esters by cultivated fibroblasts from normal individuals and patients with Schilder's disease and our measurements of the total cholesterol content of these cells. The data obtained support the concept that Schilder's disease may indeed be related to a generalized defect in cholesterol metabolism.

\section{Materials and Methods}

\section{Tissue Culture}

Fibroblasts were cultivated from three male patients with SD, all of whom had a family history of males with a degenerative neurologic disease compatible with the diagnosis of Schilder's disease. One of these patients had overt manifestations of adrenal insufficiency; a second demonstrated normal base-line function but unresponsiveness to exogenous ACTH; the third had normal base-line function with an equivocal response to ACTH administration. Cells were also cultivated from two patients with familial hypercholesterolemia (type II hyperlipoproteinemia), and from normal agematched control subjects. Cells were grown in minimum essential medium [20] supplemented with $15 \%$ fetal calf serum and were incubated at $37^{\circ}$ in an atmosphere containing $5 \% \mathrm{CO}_{2}$. At the time of our studies, all cells had undergone $5-15$ passages in tissue culture.

\section{Preparation of Labeled Medium}

$\left(4-{ }^{14} \mathrm{C}\right)$ Cholesterol and cholesteryl $\left(1-{ }^{14} \mathrm{C}\right)$ palmitate [21] were added to heat-inactivated fetal calf serum.
The labeled serum was allowed to equilibrate for several hours at $37^{\circ}$. Five percent fetal calf serum which contained labeled cholesterol or cholesterol ester was then added to minimum essential medium [20] for use in the disappearance studies while $15 \%$ fetal calf serum was added for the uptake studies. All media was counted before use. Initial activity ranged from $0.6-$ $2.0 \times 10^{4} \mathrm{cpm} / \mathrm{ml}$.

\section{Disappearance Studies}

This series of experiments was performed using both confluent cells and cells that had been subcultured 1 day before initiation of the experiment. To each 250$\mathrm{mm}$ flask of fibroblasts were added $10 \mathrm{cu} \mathrm{cm}$ medium which contained labeled cholesterol or cholesterol ester. After $24 \mathrm{hr}$ of exposure to the labeled medium, the cells were washed once with Puck's saline A solution and 10 $\mathrm{cu} \mathrm{cm}$ nonlabeled medium which contained $15 \%$ fetal calf serum were added to each flask. Each subsequent day, the medium was removed, the cells were washed once with Puck's saline, and $10 \mathrm{cu} \mathrm{cm}$ medium were again added.

Duplicate flasks of cells from each cell line were harvested on day 1 , after $24 \mathrm{hr}$ of exposure to the labeled medium, and on each subsequent day for a total of 5 days. Cells to be harvested were washed three times with normal saline and then removed from the flasks with $0.1 \%$ trypsin. The suspended cells were removed from the flasks, centrifuged, and washed twice with normal saline. The cell pellet was dissolved by heating in $0.5 \mathrm{cu} \mathrm{cm} 2.5 \mathrm{~N} \mathrm{NaOH}$. Aliquots were removed and counted in Bray's solution in a liquid scintillation counter [22]. The remainder of each sample was saved for determination of protein content [11].

\section{Uptake Studies}

Medium was removed from cells that had been subcultured 1 day previously and replaced by $20 \mathrm{cu} \mathrm{cm}$ medium containing $15 \%$ fetal calf serum and the labeled compound. Duplicate flasks of cells were harvested and counted as in the disappearance studies, at 1- or 2-day intervals for a total of 10-12 days. The medium was removed every 4 or 5 days during the course of the study and was replaced with fresh medium also containing the labeled compound.

\section{Thin Layer Chromatography}

An additional flask of each cell line was harvested on day 3 of the disappearance study for lipid analysis. The cell pellets were extracted with chloroform-meth- 
anol and thin layer chromatography was performed in accordance with the method of Gloster and Fletcher [6]. Each lipid fraction was scraped from the plate and washed several times with chloroform. The eluted samples were evaporated and counted in Bray's solution in a liquid scintillation counter [22].

\section{Determination of Total Cellular Cholesterol}

Fibroblasts were grown in medium containing $15 \%$ fetal calf serum for periods of 4,10 , and 12 days after subculture. The medium was changed at 3-4-day intervals throughout the period of study. Ten flasks of each cell line were harvested, as in the disappearance studies, and pooled into a single cell pellet. The cells were washed two times with normal saline. Each cell pellet was resuspended in distilled water to a total of $2 \mathrm{ml}$. Two-tenths milliliter was removed for protein determination [11].

To the remaining $1.8 \mathrm{ml}, 5 \mathrm{ml}$ chloroform-methanol $(2 / 1)$ were added. The sample was then allowed to stand overnight in the refrigerator. The following day, the top aqueous layer was removed and discarded. Each sample was filtered through glass filter paper and was washed in chloroform-methanol. The solvent was evaporated and the samples were redissolved in $2 \mathrm{ml}$ chloroform. One milliter was removed and saved for possible future studies. The remaining $1 \mathrm{ml}$ was evaporated. The samples were hydrolyzed with alcoholic $\mathrm{KOH}$ and extracted with ligroin. Total cholesterol was determined by the process of Abell et al. [1].

\section{Results}

\section{Disappearance of $(4-14 C)$ Cholesterol}

The results of the disappearance studies using $\left({ }^{4-14} \mathrm{C}\right)$ cholesterol are illustrated in Figure 1. The data are expressed as counts per minute per milligram protein. Duplicate flasks of each cell line were harvested each day and the values displayed represent an average of the two values obtained. The uptake of cholesterol by the end of the 24-hr period of exposure, designated day 1 , was similar in all cell lines studied. Over the following 4 days, however, cells from all three patients with Schilder's disease retained significantly greater amounts of label than did cells from control subjects. The data illustrated in Figure 1 reveal that the Schilder's cells contain an average of $80 \%$ more labeled cholesterol than do normal cells on days 2 through $5(P<$ $0.001)$. These differences are actually higher than those in a typical experiment in which only one Schilder's cell

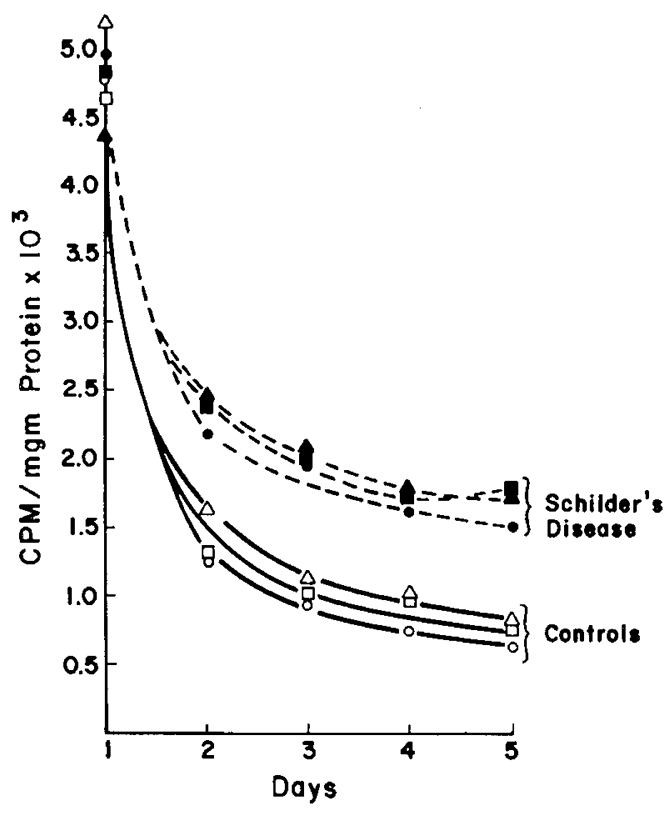

Fig. 1. Disappearance of $\left(4^{-14} \mathrm{C}\right)$ cholesterol over a 5 -day period in fibroblasts from three patients with Schilder's disease (-- ) and three control subjects $(-)$. The values are expressed in counts per minute per milligram protein.

line was studied with one or more controls. In each of the individual experiments, the Schilder's cells retained at least $25-40 \%$ more label than did the normal cells. The data presented in Figure 1 were selected for illustration because they represent the simultaneous study of the three mutant cell lines.

Cells from patients with familial hypercholesterolemia, not illustrated here, behaved as controls. Similar results were obtained when confluent or nonconfluent cells were used and when various concentrations of labeled cholesterol were added to the medium.

\section{Disappearance of Cholesteryl $\left(1-^{14} \mathrm{C}\right)$ Palmitate}

No significant differences in cholesterol ester retention could be demonstrated between $\mathrm{SD}$ fibroblasts and controls during the period of the disappearance study. Valid data was difficult to obtain, however, due to technical difficulties. The labeled cholesterol ester could not be evenly dispersed throughout the original medium, so that it was impossible to be sure that each flask of cells was exposed to the same concentration of the labeled compound. In many instances, poor duplicate values were obtained. In addition, significant quantities of label were retained by all cell lines over the 5-day course of study. It is possible that differences not obvious here might become evident if the observations were extended over longer periods of time. 


\section{Thin Layer Chromatography}

Thin layer chromatography was performed on extracts of SD and control fibroblasts on day 3 of the disappearance studies to determine whether the retained label in each cell line represented the compound administered (cholesterol or cholesterol ester) or another major lipid. The results of this study are presented in Tables I and II. The data are expressed as the percentage of the total activity of the cell preparations (counts per minute per milligram protein) present in each lipid fraction.

Essentially all of the label administered to the cell as $\left(4^{-14} \mathrm{C}\right)$ cholesterol was still detectable in the cholesterol fraction in both SD and control fibroblasts. Only 0.5-1.0\% had been converted to cholesterol esters. Of the label administered as cholesteryl (1-14C)palmitate, $88 \%$ and $90 \%$ was still located in the cholesterol ester fraction in $\mathrm{SD}$ and control fibroblasts, respectively. Small quantities of label were located in each of the other lipid fractions. No significant differences in the distribution patterns were noted between SD and control fibroblasts.

\section{Uptake of $\left(4-{ }^{14} C\right)$ Cholesterol}

The uptake of $\left(4^{-14} \mathrm{C}\right)$ cholesterol by cultivated fibroblasts is illustrated in Figure 2. The data were originally expressed as counts per minute per milligram protein in each cell sample. It is presented here as percentage of maximum control activity versus time.

Table $I$. Percentage of total counts which remain in each fraction on day 3 of cholesterol disappearance curve

\begin{tabular}{lrr}
\hline & Schilder's disease & Control \\
\hline Phospholipid & 0.5 & 1.5 \\
Cholesterol & 98.0 & 98.0 \\
Free fatty acids & 0.5 & 0.0 \\
Triglycerides & 0.0 & 0.0 \\
Cholesterol esters & 1.0 & 0.5 \\
$\quad$ Total & 100.0 & 100.0 \\
\hline
\end{tabular}

Table II. Percentage of total counts which remain in each fraction on day 3 of cholesterol ester disappearance curve

\begin{tabular}{lcc} 
& Schilder's disease & Control \\
\hline Phospholipid & 2.0 & 1.0 \\
Cholesterol & 4.0 & 3.0 \\
Free fatty acids & 2.0 & 4.0 \\
Triglycerides & 4.0 & 2.0 \\
Cholesterol esters & 88.0 & 90.0 \\
\multicolumn{1}{c}{ Total } & 100.0 & 100.0 \\
\hline
\end{tabular}

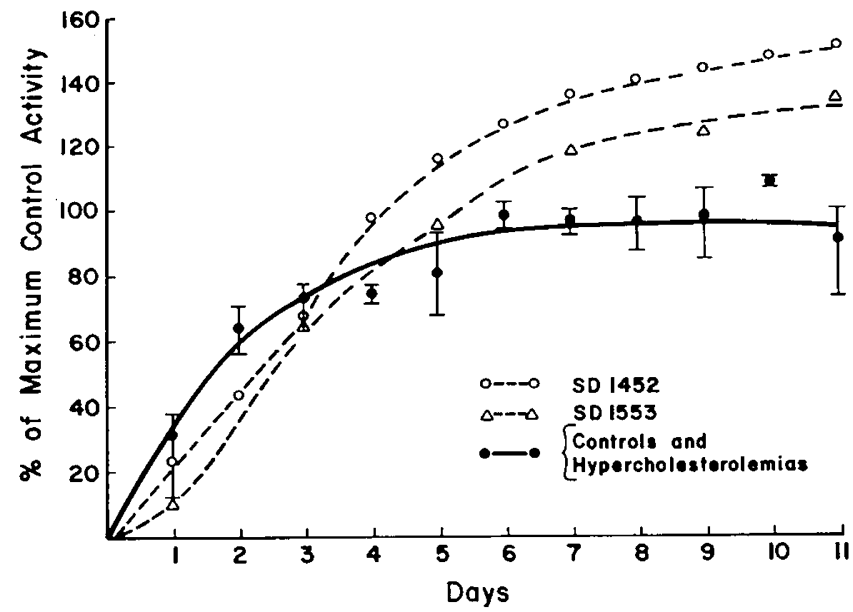

Fig. 2. Uptake of $\left(4{ }^{-14} \mathrm{C}\right)$ cholesterol in fibroblasts from two patients with Schilder's disease (- - ) and three control subjects and two patients with hypercholesterolemia $(-)$. - - composite of values for the control subjects and hypercholesterolemia patients. The data is expressed as percentage of maximum control activity versus time. Horizontal bars represent ranges of activity in control subjects and hypercholesterolemic patients.

This method of expression of the data was selected so that uptake studies performed on different occasions with various concentrations of labeled cholesterol could be consolidated and compared on a single graph. The data presented in Figure 2 represent a composite of two different studies using fibroblasts from two patients with SD, two patients with familial hypercholesterolemia, and three control subjects. Similar results were obtained using various concentrations of labeled cholesterol in the original medium.

The uptake of $\left(4^{-14} \mathrm{C}\right)$ cholesterol was similar in $\mathrm{SD}$ and control fibroblasts over the first $4-5$ days of the study period. After this time, however, the control fibroblasts reached a plateau and uptake ceased to increase appreciably. The SD fibroblasts exhibited a small but steady increase in uptake over the remaining 6-7 days of the study. On day 11, SD fibroblasts contained, on the average, 25-40\% more label than did control cells $(P<0.001)$. Fibroblasts from patients with familial hypercholesterolemia behaved as controls.

\section{Uptake of Cholesteryl $(1-14 C)$ Palmitate}

Fibroblasts from patients with Schilder's disease and from normal control subjects all continued to accumulate the cholesterol ester steadily over the 10-day period of study. This study was difficult to repeat and the data difficult to interpret because of the technical difficulties encountered in dealing with the labeled ester. 
From our limited data, however, no difference could be observed in the uptake of the ester by control and SD fibroblasts.

\section{Total Cellular Cholesterol Content}

Results of the measurements of total cholesterol in fibroblasts from one patient with $\mathrm{SD}$, two patients with familial hypercholesterolemia, and four control subjects are summarized in Table III. All values listed represent an average of two separate determinations. Four days after subculture, the cholesterol content of all cell lines was similar. After 10 days, the cholesterol content of the Schilder's cells was somewhat higher than that observed in control cells, but the difference was not statistically significant. After 12 days, however, the total cholesterol content of the SD cells was significantly higher than that of normal and hypercholesterolemic cell lines with a $P<0.01$.

\section{Discussion}

These data suggest evidence that Schilder's disease may be related to a generalized defect in cholesterol metabolism. The defect would not appear to be that of either increased cholesterol uptake from the tissue culture medium or increased endogenous synthesis of cholesterol or cholesterol esters. A likely possibility is that the defect observed in the handling of cholesterol in fibroblasts from patients with Schilder's disease is one of either intracellular metabolism or transport of cholesterol or a related compound.

Little is known of the metabolism of cholesterol, or of steroids in general, in cultivated human fibroblasts. It has previously been demonstrated that these cells have the ability to incorporate acetate into cholesterol [3] and to convert the cholesterol precursor desmosterol to cholesterol [9]. Other cell types in tissue culture are known to have the ability to esterify cholesterol, to

Table III. Total cellular cholesterol

\begin{tabular}{lccc}
\hline \multirow{2}{*}{ Cell line } & 4 Days & 10 Days & 12 Days \\
\cline { 2 - 3 } & \multicolumn{3}{c}{$\mathrm{mg} / \mathrm{mg}$ protein } \\
\hline $\begin{array}{l}\text { Schilder's disease } \\
\text { Control }\end{array}$ & 0.030 & 0.052 & 0.069 \\
1 & & & \\
2 & 0.040 & 0.047 & 0.048 \\
3 & 0.036 & 0.045 & 0.038 \\
4 & 0.038 & 0.042 & 0.042 \\
Hypercholesterolemia & & 0.046 & \\
1 & & & \\
2 & 0.035 & & 0.051 \\
Mean of Controls & 0.038 & & 0.053 \\
& 0.038 & 0.045 & 0.043 \\
\hline
\end{tabular}

hydrolyze cholesterol esters to free cholesterol and fatty acids, to cleave the first carbon atom from the fatty acid of cholesterol esters to yield $\mathrm{CO}_{2}$, and to take up both cholesterol and cholesterol esters from the tissue culture medium but to release only free cholesterol [15].

At the present time, no data is available with regard to the ability of cultivated human fibroblasts to synthesize other sterols from cholesterol. It is possible that Schilder's disease might be related to a metabolic block involving an intermediate in sterol metabolism rather than cholesterol itself. In this case, the label derived from cholesterol might accumulate within the cells either as the sterol or as cholesterol. An alternative hypothesis is that the retention and accumulation of labeled cholesterol observed in Schilder's fibroblasts is related to a nonspecific defect in membrane turnover or transport. Cholesterol is an important constituent of cell membranes and a defect in membrane structure or degradation might be reflected by a relatively normal rate of incorporation of the labeled compound into membrane structures but delayed release. Likewise, a defect in membrane transport mechanisms might be reflected by difficulty in the extrusion of cholesterol and of other compounds from the cell.

It is clear that further studies of cholesterol transport and metabolism in normal fibroblasts, fibroblasts of patients with Schilder's disease, and fibroblasts from patients with other degenerative neurologic diseases are essential to the further interpretation of the data presented here. Such studies may help to elucidate the basic biochemical defect in this disorder.

\section{Summary}

The uptake and disappearance of labeled cholesterol and cholesterol esters has been studied in cultivated fibroblasts from patients with Schilder's disease, patients with familial hypercholesterolemia, and normal control subjects. The initial uptake of cholesterol was similar in control and Schilder's fibroblasts but, on continuous prolonged exposure to the labeled compound, Schilder's fibroblasts accumulated significantly greater amounts of label than did control cells. When, after a 24-hr exposure to labeled cholesterol, the labeled compound was removed from the tissue culture medium, fibroblasts from patients with Schilder's disease retained significantly greater amounts of label than did control cells. The retained label remained in the cholesterol fraction on thin layer chromatography. Measurements of total cholesterol in fibroblasts after pro. 
longed periods in tissue culture revealed an absolute increase in the total cholesterol content of Schilder's cells as compared with normal cells. No significant differences were observed between Schilder's and normal cells in the uptake and disappearance of cholesterol esters. Fibroblasts from patients with familial hypercholesterolemia behaved as controls in all studies.

These data indicate clearly that Schilder's disease may be related to a generalized defect in cholesterol metabolism or transport.

\section{References and Notes}

1. Abell, L. L., Levy, B. B., Brodie, B. B., And Kendall, F. E.: A simplified method for the estimation of total cholesterol in serum and demonstration of its specificity. J. Biol. Chem., 195: 357 (1952).

2. AdAMs, R. D., AND KubiK, C. S.: The morbid anatomy of the demyelinative diseases. Amer. J. Med., 12: 510 (1952).

3. Avigan, J., Williams, C. D., AND Blass, J. P.: Regulation of sterol synthesis in human skin fibroblast cultures. Biochim. Biophys. Acta, 218: 381 (1970).

4. ETo, Y., AND SUzuKI, K.: Fatty acid composition of cholesterol esters in brains of patients with Schilder's disease, $\mathrm{G}_{\mathrm{MI}^{-}}$ gangliosidosis and Tay-Sachs disease, and its possible relationship to the $\beta$-position fatty acids of lecithin. J. Neurochem., 18: 1007 (1971).

5. Forsyth, C. C., Forbes, M., And Cumings, J. N.: Adrenocortical atrophy and diffuse cerebral sclerosis. Arch. Dis. Childhood, 46: 273 (1971).

6. Gloster, J., AND Fletcher, R. F.: Quantitative analysis of serum lipids with thin-layer chromatography. Clin. Chim. Acta, 13: 235 (1966).

7. Hokfnagel, D., Brun, A., Ingbar, S. H., and Goldman, H.: Addison's disease and diffuse cerebral sclerosis. J. Neurol. Neurosurg. Psychiat., 30: 56 (1967).

8. Hoeffnagel, D., Van Den Noort, S., and Ingbar, S. H.: Diffuse cerebral sclerosis with endocrine abnormalities in young males. Brain, $L X X X V: 553$ (1962).

9. Holmes, R., Helms, J., AND Mercer, G.: Cholesterol requirement of primary diploid human fibroblasts. J. Cell Biol., 42: $262(1969)$.

10. Kovacs, K., Blascheck, J. A., Yeghiayan, E., Hatakeyama, S., aND Gardell, C.: Adrenocortical lipid hyperplasia induced in rats by aniline. Amer. J. Pathol., 62: 17 (1971).

11. Lowry, O. H., Rosebrough, N. J., FarR, A. L., and Randall,
R. J.: Protein measurement with the Folin phenol reagent. J. Biol. Chem., 193: 265 (1951).

12. Marshall, W. C., Ockenden, B. G., Fosbrooke, A. S., and Cumings, J. N.: Wolman's disease: a rare lipidosis with adrenal calcification. Arch. Dis. Childhood, 44:331 (1969).

13. Menkes, J. H., and Eviatar, L.: Diffuse sclerosis and Addison's disease: evidence for the presence of an abnormal sterol in white matter (Abstract). Pediat. Res., 3: 359 (1969).

14. Poser, C. M., and van Bogaert, L.: Natural history of evolution of the concept of Schilder's diffuse sclerosis. Acta Psychiat. Neurol. Scand., 31: 285 (1956).

15. Rothblat, G. H., Hartzell, R., Mialhe, H., and KritchevSKY, D.: Cholesterol metabolism in tissue culture cells. In: G. H. Rothblat and D. Kritchevsky: Lipid Metabolism in Tissue Culture Cells, pp. 129-153 (Wistar Institute Press, Philadelphia, 1967).

16. Schaumberg, H. H., Richardson, E. P., Johnson, P. C., Cohen, R. B., Powers, J. M., And Raine, C. S.: Schilder's disease. Arch. Neurol., 27: 458 (1972).

17. SUZUKI, N., AND GRover, W. D.: Ultrastructural and biochemical studies of Schilder's disease: I. Ultrastructure. J. Neuropathol. Exp. Neurol., 29: 392 (1970).

18. Turkington, R. W., and Stempfel, R. S., JR.: Adrenocortical atrophy and diffuse cerebral sclerosis (Addison-Schilder's disease). J. Pediat., 69: 406 (1966).

19. VICK, N. A., AND MOORE, R. Y.: Diffuse sclerosis with adrenal insufficiency. Neurology, 18: 1066 (1968).

20. Grand Island Biological Co., Grand Island, N. Y.

21. Amersham-Searle Corp., Arlington Heights, Ill.

22. Mark II liquid scintillation counter, Nuclear Chicago Corp., Des Plaines, Ill.

23. We are grateful to Drs. Maria Gumbinas and Marianne Larsen for allowing us to study their patient and to Drs. Kurt Hirschhorn and Michel Philippart for supplying us with fibroblasts from patients with Schilder's disease. Drs. Avedis Khachadurian and Frank Kawahara performed the thin layer chromatography and measurements of total cellular cholesterol.

24. This work was supported by Grants nos. HD 00036 and HD 04252, The National Institutes of Health, by The National Foundation-March of Dimes, and by The Spastic Paralysis Research Foundation. Dr. H. L. Nadler is the Irene Heinz Given and John La Porte Given Research Professor of Pediatrics.

25. Requests for reprints should be addressed to: H. L. NAdLER, M.D., Children's Memorial Hospital, 2300 Children's Plaza, Chicago, Ill. 60614 (USA).

26. Accepted for publication October 17, 1973. 